\title{
INFLUENCE OF PHARMACIST-DOCTOR COMMUNICATION ON PEDIATRIC ANTIBIOTIC PRESCRIPTIONS
}

\author{
DIANY ASTUTI ${ }^{1}$, RETNOSARI ANDRAJATI ${ }^{1 *}$, SUDIBYO SUPARDI $^{2}$
}

${ }^{1}$ Department of Clinical Pharmacy, Faculty of Pharmacy, Universitas Indonesia, Depok, Indonesia. ${ }^{2}$ Institution of Health Research and Development, Ministry of Health Republic of Indonesia, Indonesia. Email: andrajati@farmasi.ui.ac.id

Received: 21 April 2017, Revised and Accepted: 13 July 2017

\section{ABSTRACT}

Objectives: The purpose of the research was to describe drug-related issues and assess the effect of communication between pharmacists and physicians on decreased prescription drug-related problems in pediatric antibiotics.

Methods: Intervention was carried out in the form of pharmacist discussing information-related issues about drugs with physicians prescribing antibiotics to pediatric patients. The research sample included 338 prescription sheets on pediatric outpatient use of antibiotics, and a prospective design was used. Analysis of the data was carried out in the form of a frequency distribution and bivariate analysis Wilcoxon test.

Results: The results showed that drug-related problems were recorded on $62.22 \%$ of the 338 prescription sheets. The biggest problems that occurred had to do with the effectiveness of therapy (P1; 79.3\%) and cost issues (P1; 20.7\%). In terms of P1, drug-related problems involved excessive frequency of drug administration (80.1\%), inappropriate drug dose selection (17.2\%), drug interactions (9.6\%), inadequate drug selection (12.2\%), a subtherapeutic dose (4.1\%), and excessive drug dose (0.5\%). For P1, therapy cost problems were a lack of indication for the drug (84.1\%) and improper drug selection (15.9\%).

Conclusions: The influence of pharmacist-physician communication concerning the reduction of drug-related problems for pediatric outpatient prescriptions was statistically significant $(\mathrm{p}<0.05)$. Ultimately, communication between pharmacists and physicians can reduce drug-related problems by $22.9 \%$.

Keywords: Prescription, Drug-related problems, Hospital, Pediatric.

(C) 2017 The Authors. Published by Innovare Academic Sciences Pvt Ltd. This is an open access article under the CC BY license (http://creativecommons. org/licenses/by/4. 0/) DOI: http://dx.doi.org/10.22159/ajpcr.2017.v10s5.23093

\section{INTRODUCTION}

Drug-related problems are a term that describes every event that has a potential to influence the result of a therapy given to a patient. Drugrelated problems can happen at any stage of therapy, from writing a prescription to the drug's administration. The limited knowledge about the medical therapy on the part of patients/family could also influence the number of drug-related problems [1]. Pediatric patients (aged 1-12 years) have different needs in the pharmaceutical context. This is related to the physiological changes of this group caused by the growth and development process. The development of pediatric biological processes could influence drug metabolism and excretion; thus, adjustments of the dosage and therapy interval are needed for pediatric patients [2].

Drug-related problems that may arise in pediatric and adolescent patients include side effects, drug interactions, duplication of therapy, unfit choice of drugs, subtherapeutic doses, overdose/toxic dose, drug consumption without indication, and patient noncompliance. Pediatric patients have a high probability of adverse side effects. The most commonly identified therapies with adverse side effect for pediatric patients are antimicrobial drugs (especially, penicillin, cephalosporin, and vancomycin), anticonvulsants, narcotics, anti-emetics, and contrast media. Some therapies are even contraindicated for pediatric patients. In Indonesia, the use of antibiotic therapy for pediatric patients had some weaknesses, particularly in the case management of a cough or difficult breathing where the second-line antibiotics were used without the evidence of need [3]. The irrational utilization, such as overuse, of antibiotics has an important role in bacterial resistance. Such resistance causes morbidity and mortality, as well as additional cost to cure the adverse effect. Data from the World Health Organization (WHO) show that 440,000 new cases of multidrug-resistant tuberculosis (TB) arise every year, causing at least 150,000 deaths.

Pediatric patients' level of compliance with therapy also contributes to the number of drug-related problems. These patients can be non-cooperative during therapy, especially in terms of drug administration, as the administration of drugs may be painful or uncomfortable and drugs are usually bitter; thus, young patients may refuse to take them. The behavior and knowledge of parents or drug administrators concerning the therapy could influence the success of administration. Complex or difficult therapy administration, as well as therapy involving lifestyle changes, could have a great influence on the number of drug-related problems. The pharmacist's expertise as the provider of pharmaceutical services in identifying, preventing, and solving drug-related problems is required to support the achievement of a given therapy. However, pharmacists' identification of drugrelated problems cannot support the achievement of therapy if they are not followed up to ensure the problems are resolved. Thus, the resolution of drug-related problems often depends on communication between the pharmacist and the doctor prescribing the therapy, the patient/patient's family, or other medical staff involved in patient care. Communication between pharmacists and doctors is required to decrease the number of drug-related problems. The shift in pharmaceutical services to patient-oriented services has underscored the importance of pharmacist's ability to communicate with other medical staffs to achieve successful therapy. This has resulted in a new relationship between pharmacists and doctors, as both parties must foster communication and a team effort to administer the prescribed treatment [4]. The purpose of the research was to describe drug-related issues and assess the effect of communication between 
pharmacists and physicians on decreased prescription drug-related problems in pediatric antibiotics.

\section{METHODS}

The study was conducted using a pre-experimental method and a prospective design. It was carried out from early August to late September 2015 by reviewing prescriptions from the pediatric clinic at Proklamasi Hospital in a suburb Indonesia. The study was conducted on pediatric patients who received prescriptions containing antibiotics. Every prescription was assessed to identify drug-related problems; problems that were identified were brought to the attention of the doctor by the pharmacist with the appropriate recommendations.

Data collection was performed through the direct assessment of prescriptions that contained antibiotics given to pediatric patients during service in the pediatric clinic. Secondary data needed in the assessment process were obtained from the patients' medical records. All information related to drugs were recorded and communicated promptly with the doctor with suitable recommendations. The results of communication were recorded and recapitulated to be analyzed using univariate and bivariate analysis. The test was done for all paired data before and after communication using the Wilcoxon test, and a confidence interval of $95 \%$ was applied. The results of this study are presented descriptively and analytically.

The study involved every pediatric outpatient. The samples were prescriptions at Proklamasi Hospital that met the inclusion criteria, which were prescriptions and medical records of pediatric outpatients diagnosed with infection and experiencing drug-related problems were included. Prescriptions and medical records of neonatal patients were excluded from the study.

\section{RESULTS}

\section{Characteristics of patient demographic data}

Table 1 showed the demographic data of patients with infection based on gender showed that 177 boys (52.2\%) and 162 girls (47.8\%) met the inclusion criteria. In terms of the age groups of pediatric outpatients, 60 patients $(17.7 \%)$ were infants aged 1-11 months, $208(61.4 \%)$ were toddlers aged $12-59$ months, and $71(20.9 \%)$ were children aged 6-12 years.

\section{Description of diseases}

The pediatric outpatient clinic at Proklamasi Hospital treated patients with various diseases and different diagnoses in August-September 2015. Antibiotics were utilized to treat the 10 most common diseases, including TB and respiratory infection. Infection had the highest diagnostic prevalence during the study period. The complete data are presented in Table 2.

The most treated infectious disease was TB, at $40.42 \%$, followed by respiratory infection, at $27.43 \%$. The high prevalence of TB in pediatric patients paralleled the health profile data for Karawang, which showed an increase of TB patients in 2014 compared with 2011. In data from the National Basic Health Survey 2013, West Java had the highest number of TB cases in Indonesia. In the pediatric population, most children with TB were 1-4 years of age. Respiratory infection (27.43\%) showed the second highest prevalence concerning pediatric infectious disease (PID) at the pediatric outpatient clinic Proklamasi Hospital. The number of patients with respiratory infection increased drastically in 2012 (33.4\%) compared with 2011. The data showed a decrease in the prevalence of respiratory infection due to pneumonia in 2014. The prevalence of respiratory infection in 2013 (25.0\%) exhibited only a slight change from the data for 2013, which reported the rate as $25.5 \%$. Most of those with respiratory infection were children aged 1-4 years (Riskesdas, 2013).

\section{Description of prescribed medicine}

Drug utilization at the pediatric outpatient clinic showed that 39 types of medicine were used. The drug utilization was differentiated between
Table 1: Frequency distribution of patients' characteristics

\begin{tabular}{ll}
\hline Demographic characteristic & n (\%) \\
\hline Gender & \\
Male & $177(52.2)$ \\
Female & $162(47.8)$ \\
Age (months) & \\
$1-11$ & $60(17.7)$ \\
$12-59$ & $208(61.4)$ \\
$60-144$ & $71(20.9)$ \\
\hline
\end{tabular}

Table 2: Distribution of infectious diseases at the pediatric outpatient clinic

\begin{tabular}{ll}
\hline Diagnosis & Frequency total (\%) \\
\hline TB & $137(40.41)$ \\
Respiratory infection & $93(27.43)$ \\
Common cold & $27(7.96)$ \\
Typhoid & $25(7.37)$ \\
Acute gastroenteritis & $17(5.01)$ \\
Fever & $10(2.95)$ \\
Pharyngitis & $6(1.77)$ \\
Dermatitis & $6(1.77)$ \\
Convulsions & $3(0.88)$ \\
Other & $15(4.42)$ \\
Total & $314(100)$ \\
\hline
\end{tabular}

TB: Tuberculosis

antibiotic and non-antibiotic drugs. Antibiotic utilization during the study period at the pediatric outpatient clinic at Proklamasi Hospital included nine types of antibiotics. The groups of most frequently utilized antibiotics were cephalosporin, penicillin, and anti-TB medication. In antibiotic group, the most utilized antibiotics were cefadroxil (33.8\%) and rifampicin $(27.2 \%)$. In this analysis, vitamins, supplements, and drugs in the form of creams, solutions, eardrops, and eye drops were not included in the analysis. Patients with infectious disease were also given non-antibiotic complement drugs. Such drugs were generally administered to reduce patients' symptoms.

Identification of 338 prescriptions with antibiotic utilization showed 208 drug-related problems. The biggest problem in pediatric patients' prescription concerned the effectiveness of therapy (97.3\%) and cost of therapy $(20.7 \%)$. In this study, the effectiveness of therapy was connected to a suboptimal therapy effect.

In identifying the problem groups for drug-related problems using the Pharmaceutical Care Network Europe (PCNE) v.6.2 instruments, four problem groups were delineated. For the group of adverse side effects in therapy, there was a minor interaction between cefadroxil and Vitamin B1. The reaction was a decrease in the activity of Vitamin B1. This would not be significant if Vitamin B was given orally. In this study, the administration of cefadroxil with Vitamin B1 was not categorized as a problem.

Every problem group in the identification of drug-related problems with PCNE v. 6.2 could has been caused by more than one source of drugrelated problems. In this study, it was identified that there were 240 problems with pediatric patients' prescriptions, including 196 cases of non-optimal effectiveness of therapy and 44 cases of the cost of therapy. In this study, non-optimal effectiveness of therapy may have been caused by several factors. The identified factors were the inaccurate choice of drugs, subtherapeutic dose, overdose, too long a duration of drug administration, and too short a duration of drug administration.

From the 196 cases, the main causes of non-optimal effect of therapy were too high a frequency of drug administration (80.1\%), incorrect choice of drugs $(12.2 \%)$, and subtherapeutic dose of drugs $(4.1 \%)$. Other causes recorded were too low a duration of therapy (3.1\%) and 
overdose of drugs (0.5\%). The study by Abrogoua et al. (2015) on pediatric outpatients who received antibiotics at a hospital in France showed a non-optimal drug administration (88.9\%) and subtherapeutic dose of drugs (11.1\%) as the most frequent drug-related problems. The study conducted had similar patterns, whereby the ineffectiveness of therapy caused drug-related problems, but in the present study, the main cause was too high a frequency of drug administration (80.1\%). From 157 cases, the drugs that caused the too high a frequency of drug administration were cefadroxil (99.4\%) and rifampicin (0.6\%). The data on these antibiotics are presented on Table 3.

In the cefadroxil prescription, medication was administered 3 times a day. Taketomo et al. [5] stated that the rule for cefadroxil administration is $30 \mathrm{mg} / \mathrm{kg}$ bodyweight each dose every $12 \mathrm{hrs}$. Moreover, British Pharmacopoeia for Children [6] also delineates the same rule for cefadroxil administration. The proportion of drug-related problems related antibiotic utilization in the pediatric outpatient clinic at Proklamasi Hospital due to the inappropriate choice of drugs was $12.2 \%$. Cefadroxil (95.8\%) and thiamphenicol (4.2\%) were the drugs in this category with 24 total cases. In this study, cefadroxil was inappropriately chosen with a diagnosis of the common cold (88.2\%). Drug-related problem also occurred in terms of selection of antibiotics and antivirals. In this case, the administration of an adequate antiviral was sufficient. The selection of thiamphenicol for the common cold was also categorized as an inappropriate choice of drugs. Thiamphenicol is used for cases involving bacterial infection, such as typhoid. Thiamphenicol was only prescribed in one case in this study.

The common cold is an acute infectious disease of the upper respiratory system caused by rhinovirus. The administration of antibiotics for the common cold is not effective because the target is not a microbe [7]. Therapy with sufficient liquid could ease the symptoms caused by the common cold [8]. The administration of drugs at a subtherapeutic dose could occur in adults as well as pediatric patients. Safety is a key factor in determining doses for pediatric patients. For children, the rule that is generally used is based on the unit milligram per kilogram of body weight. Thus, it is necessary to know the patient's body weight.

In this study of drug-related problems concerning antibiotic administration at the pediatric outpatient clinic at Proklamasi Hospital, it was found that a subtherapeutic dose $(4.1 \%)$ was one of the reasons for a suboptimal therapy effect. Out of 6 cases, cefixime (50.0\%) was most frequently associated with a subtherapeutic dose. The other antibiotics in this group were rifampicin (33.3\%) and isoniazid (16.7\%). Abrogoua et al. $[9,10]$ conducted a study on pharmacist intervention in outpatient prescriptions and found that the proportion of drug-related problems involving a subtherapeutic dose was $11.1 \%$; the antibiotic that was most frequently involved was amoxiclav (61.9\%).

To identify the drugs classified in the subtherapeutic dose category, a comparison was conducted between the prescribed drugs and the literature. The given dose of drugs was considered too low if the

Table 3: Distribution of antibiotic utilization at the pediatric outpatient clinic

\begin{tabular}{ll}
\hline Drug names & Frequency \\
\cline { 2 - 2 } & $\mathbf{n}(\%)$ \\
\hline Cefadroxil & $163(33.8)$ \\
Rifampicin & $131(27.2)$ \\
Isoniazid & $96(19.9)$ \\
Pyrazinamide & $50(10.4)$ \\
Cefixime & $29(6.1)$ \\
Acyclovir & $6(1.2)$ \\
Amoxicillin & $5(1.1)$ \\
Metronidazole & $1(0.2)$ \\
Thiamphenicol & $1(0.2)$ \\
Total & $482(100)$ \\
\hline
\end{tabular}

deviation was at least 5\% lower than the accepted lowest dose with the result of calculation of dose per kilogram of body weight. The final drug-related problems in the suboptimal therapy effect group were caused by too low a duration of therapy (3.1\%) and overdose $(0.5 \%)$. An antibiotic that was classified into this category was cefadroxil. This drug was administered for pharyngitis therapy for 4 days. Based on Taketomo et al. [5], the appropriate cefadroxil therapy for pharyngitis should be 10 days.

The pharmacist communicated with the doctor to overcome the drugrelated problem. Communication was performed by giving information about the duration of cefadroxil administration after the reconfirmation of cefadroxil therapy, which was carried out by telephone. The communication did not result in any changes to the duration of medication given to the patient because the patient was expected to come back for a checkup after finishing the medicine. Drug-related problems in the overdose category occurred with the administration of cefixime. The alteration of therapy was accepted for half of the drugrelated problems. The other categories of drug-related problems that were identified in this study involved the cost of therapy (20.7\%). In this group, the reasons for the drug-related problem were the inappropriate choice of drugs and lack of indication for the prescription.

Out of 44 cases, the reasons for drug-related problems concerning therapy cost were no indication (84.1\%) and an inappropriate choice of drugs (15.9\%). The unsuitable selection of drugs occurred in the administration of cefadroxil for diarrhea. Based on the WHO [11], the treatment for diarrhea in pediatric patient does not include antibiotics. The therapy should be administration of fluid to prevent dehydration and supportive zinc therapy. The administration of antibiotics was supposed to treat diarrhea that could lead to dysentery or cholera. The antibiotic given was based on the type of pathogenic microbes. The other reason for drug-related problems concerning therapy cost was a lack of indication (84.1\%) for antibiotic administration. From 37 cases, the administration of cefadroxil (97.3\%) and thiamphenicol (2.7\%) was the reason for no indication for therapy in the cost of therapy problem group. The reasons for administration of these antibiotics were similar; they were used to treat the common cold. The inappropriate choice of drugs in the suboptimal therapy effect group also influenced the cost of therapy. The inappropriate choice of drugs led to a higher cost of therapy

\section{The effect of pharmacist-doctor communication on decreasing} drug-related problems

This study included all prescriptions for pediatric outpatients during the study period. The identification of drug-related problems was conducted for 338 prescriptions that involved the administration of antibiotic/antimicrobial drugs and 278 non-antibiotic prescriptions. There were 240 cases of drug-related problems concerning antibiotic prescriptions at the pediatric outpatient clinic at Proklamasi Hospital. Communication by the pharmacists with the doctors resulted in a decrease in drug-related problem cases. There were various numbers of drug-related problems related to each prescription. When drugrelated problems were identified, the pharmacist intervened by suggesting replacement/improvement of the prescription through direct communication with the doctor.

In this study, a test was conducted to identify the significance of pharmacist-doctor communication in decreasing the drug-related problems of pediatric patients with infectious diseases at Proklamasi Hospital. The test was conducted using bivariate analysis involving the Wilcoxon test for paired samples. This test was chosen because the data obtained were paired data, and the data involved the differences in scores of drug-related problem before and after communication were performed. The data were also non-parametric data. The distribution of data was not normal. The test result showed a significance value of 0.000 . This was far below the set al.ha significance of $5 \%$. Based on the test result, it was concluded that there was a significant difference in the communication in terms of the number of problems before and 
after the intervention. Thus, it could be concluded that pharmacistdoctor communication could have a significant effect in decreasing the drug-related problems of pediatric patients with infectious diseases. The reduction in the prevalence of drug-related problem after communication with the doctor was $22.9 \%$.

\section{DISCUSSION}

Due to the potential for drug-related problems, clinical pharmacy activities have an important role in the success of patient therapy. Skills in PID and antibiotic management are of critical importance in hospitals [12]. The intervention was carried out in the form of pharmacist discussing information-related issues about drugs with physicians prescribing antibiotics to pediatric patients. The current data showed that pediatric prescriptions involving antibiotics were mainly prescribed to male patients (52.2\%), toddlers aged 12-59 months $(61.4 \%)$, and TB patients (40.41\%). Drug-related problems were found on $62.2 \%$ of pediatric patients' prescriptions containing antibiotics, and the problems involved the effectiveness of therapy (79.3\%) and cost of therapy $(20.7 \%)$. The reasons for problems in pediatric prescription that contained antibiotics related to the effectiveness of therapy had to do with too high a frequency of drug $(80.1 \%)$, inappropriate choice of drugs $(12.2 \%)$, subtherapeutic dose $(4.1 \%)$, and overdose $(0.5 \%)$. In the cost of therapy group, the reasons were no indication for therapy (84.1\%) and inappropriate choice of drugs (15.9\%).

The pharmacists' communication with doctors had to do with the frequency of drug administration. The result of communication was alteration of the frequency of drugs administration to 3 times a day. This alteration of frequency was generally approved for older pediatric patients (not infants/toddlers). The alteration of dose for cefadroxil was based on the consideration of the unsuccessful administration of drugs, mostly because they were thrown up by patients. To overcome the problem, administration of cefadroxil was changed to 3 times a day so that the appropriate level of absorption could be achieved. Cunha [7] classified cefadroxil into the group of time-dependent antibiotics. The frequency of drug administration can be increased to enhance the effectiveness of time-dependent antibiotics. Cefadroxil can maintain the concentration above the minimum-resistant concentration for a specific duration. The maximum dose of cefadroxil in this study was not exceeded, but the level of drugs for one administration was a subtherapeutic dose. Drug-related problems in antibiotic administration at the pediatric outpatient clinic at Proklamasi Hospital was also influenced by the inappropriate choice of drugs. An unsuitable choice could influence the result of therapy in terms of underactivity of therapy, longer duration of therapy, or even adverse effects.

The high utilization of cefadroxil was found in cases of respiratory infection and the common cold. The utilization of antibiotics for common cold was not appropriate because the disease is not caused by bacteria. Cefadroxil was also used to treat patient with fever. Fever usually represents an ongoing infection, so in this case, further examination should has been done to determine the diagnosis. The utilization of antibiotics for patients with non-infectious disease was rationalized according to the potential that the disease could lead to infectious disease. This caused drug-related problems in terms of the prescription of unnecessary/non-indicated drugs. Further examination should have been carried out in these cases so that additional therapy costs could be avoided. The other most utilized drugs were drugs in anti-TB group. This paralleled the diagnosis of most treated diseases at the pediatric outpatient clinic at Proklamasi Hospital, which were TB and respiratory infection. There were few problems identified in terms of dose and duration of drug consumption for anti-TB drug, as this kind of disease follows a program for controlling TB implemented by the Ministry of Health. Moreover, the prescribed drugs involved drugs with fixed combinations. The problems with TB patients mostly related to the patient and patient's family $[13,14]$. It was necessary to give clear, detailed information to the patient. However, there were some mistakes in the medication consumed by patients because they did not understand the instructions. It is an uncomfortable situation when the information given can potentially cause such a problem. Information needs to be given to TB patients more carefully and exhaustively than it does for any other patients. However, the long queues and parents/family wanting to go home because the patients were out of sorts may have resulted in distracting family members when they should have directed their full attention to receiving the information. This study included some limitations. Clinical evaluation of the patients was not conducted, so the result of the therapy could not be definitively identified. There was a psychological barrier concerning pharmacists' contacting medical staff and patients directly in terms of clinical pharmacy application. Despite the limitations, the data presented could be an initial precursor for implementing clinical pharmacy practice at Proklamasi Hospital in the future. The pharmaceutical department could determine the program for the next period based on descriptive data in this study concerning the medicine administrated at Proklamasi Hospital.

\section{CONCLUSION}

Pharmacist-doctor communication significantly decreased the number of drug-related problems. The decrease in drug-related problems for pediatric patient prescriptions was $22.9 \%$.

\section{REFERENCES}

1. Adusumili P, Adepu R. Drug related problems: An over of various classification. Asian J Pharm Clin Res 2014;7(4):8-10.

2. Seyberth HW, Rane A, Schwab M. Pediatric Clinical Pharmacology. New York: Springer Heidelberg; 2011.

3. Sidik NA, Lazuardi L, Agung FH, Pritasari K, Roespandi H, Setiawan T, et al. Assessment of the quality of hospital care for children in Indonesia. Trop Med Int Health 2013;18(4):407-15.

4. Siregar CJ. Farmasi Klinik Teori dan Penerapan. Jakarta: Penerbit Buku Kedokteran EGC; 2004.

5. Taketomo CK, Hodding JH, Donna KM. Pediatric Dosage Handbook. $17^{\text {th }}$ ed. USA: American Pharmacist Association, Lexi-Comb; 2010

6. British Pharmacopeia Commission. British Pharmacopeia. London: Stationery Office; 2012.

7. Cunha AB. Antibiotic Essentials. $14^{\text {th }}$ ed. Bangladesh: Jaypee Brothers Medical Publishers; 2015.

8. Simasek M, Blandino DA. Treatment of the common cold. Am Fam Physician 2007;75(4):515-20.

9. Abrogoua DP, Koffi NO, Doffou E. Pharmacist interventions on antibiotic prescriptions in outpatient pediatric unit in a teaching hospital of Côte d'Ivoire. Ann Pharm Fr 2016;74(5):380-8.

10. Abrogoua DP, Békégnran CP, Gro BM, Doffou E, Folquet MA. Assessment of a clinical pharmacy activity in a pediatric inpatient department in cote D'ivoire. J Basic Clin Pharm 2016;8(1):15-9.

11. World Health Organization. The Treatment of Diarrhoea: A Manual for Physicians and Other Senior Health Workers. Geneva: WHO; 2005.

12. Angoulvant F, Dubos F, Cohen R; Groupe de pathologie infectieuse pédiatrique, Martinot A. Nationwide survey on pediatric infectious disease organisation and antibiotic management in French hospitals. Arch Pediatr 2015;22(5):485-90.

13. Tasnim S, Rahman A, Hoque FM. Patient's knowledge and attitude towards tuberculosis in an Urban setting. Pulm Med 2012;2012:352850.

14. Jin J, Sklar GE, Min Sen Oh V, Chuen Li S. Factors affecting therapeutic compliance: A review from the patient's perspective. Ther Clin Risk Manag 2008;4(1):269-86. 